\title{
INVESTIGATION OF SIMULTANEOUS CATION- $\pi$ AND $\pi-\pi$ STACKING INTERACTIONS ON GRAPHENE AND SOME BENT GRAPHENES AS CURVED SURFACES OF CARBON NANOHORNS
}

\begin{abstract}
P. Karimi
Computational quantum chemistry methods are used to study simultaneous cation- $\pi$ and $\pi-\pi$ stacking interactions with a graphene sheet and on the inner and outer faces of some bent graphenes as curved surfaces of carbon nanohorns (CNHs). Structural parameters and energy data of ternary benzene-graphene$\mathrm{Na}^{+}$and benzene-bent graphene- $\mathrm{Na}^{+}$complexes are studied. Also, effects of charge transfer and aromaticity are estimated to determine how changes in the structure influences the above interactions. The results indicate that the graphene curvature leads to structural changes affecting simultaneous interactions of the $\mathrm{Na}^{+}$cation and benzene with bent graphenes. Also, the results show that although $\pi-\pi$ stacking is a weak interaction, it can manipulate the order of binding energies in complexes involving both mentioned interactions and affect drug delivery abilities of these systems.
\end{abstract}

DOI: $10.1134 / \mathrm{S} 0022476618050116$

Keywords: bent graphene, carbon nanohorns, cation- $\pi, \pi-\pi$ stacking, aromaticity.

\section{INTRODUCTION}

Carbon nanohorns (CNHs) are a kind of nanomaterials similar to single-walled carbon nanotubes (SWCNTs) made of graphene sheets $[1,2]$. However, these materials differ significantly from SWCNTs because they have long cone-shaped tips with cone angles of about $20^{\circ}$ and large tube diameters of 2-5 $\mathrm{nm}$. They indicate good intrinsic properties such as a large specific surface area [3], high conductivity [4], and high ability for dispersion [5]. Thus, CNHs have useful applications such as gas adsorption [6, 7], capacitors [8-10], sensing [11], and drug delivery carriers [12-14].

CNHs could be oxidized to generate functional groups for biomodifications whose oxygenated groups react with protein bovine serum albumin (BSA) to create highly dispersed bio-conjugates [15]. Also, zinc phthalocyanine could be attached to oxidized CNHs through $\pi-\pi$ stacking interactions and then functionalized with BSA. Results of photo excitations reveal charge transfer from zinc phthalocyanine to oxidized CNHs and highlight the utilization of these materials in photobiology [16]. Also, CNHs could be decorated by gold nanoparticles to form water-soluble nanohybrid colloids which are biocompatible and have biomedical applications [17]. Moreover, soluble and biocompatible nanohybrids made of assembled liposomes onto CNHs have valuable medicinal applications, e.g. in vaccine development and drug delivery [18].

Department of Chemistry, Faculty of Science, University of Zabol, Zabol, Iran; pkarimi@uoz.ac.ir. The text was submitted by the authors in English. Zhurnal Strukturnoi Khimii, Vol. 59, No. 5, pp. 1137-1143, June-July, 2018. Original article submitted March 12, 2017. 
Structure and size of CNHs make them potential vehicles for intracellular delivery. In fact, they can be isolated by a copolymer and these nontoxic modified CNHs are good vehicles for intracellular delivery [19]. In vitro and in vivo experiments suggest that $\mathrm{CNHs}$ have negative mutagenic possibilities and are not carcinogenic [20].

$\mathrm{CNHs}$ are good candidates for medical applications. For instance, CNHs have applications in antitumor therapy and oxidized CNHs entrap anticancer agent cisplatin that is released from $\mathrm{CNHs}$ in aqueous environments. Released cisplatin plays an effective role in inhibiting the growth of human lung cancer cells [21, 22]. Furthermore, vancomycin hydrochloride (VCM) can incorporate into oxidized CNHs, which leads to controlled release of VCM in prolonged periods [23]. Indeed, adsorption of polyethelene glycol-doxorubicin conjugate onto CNHs improves their dispersibility in water and makes them valuable drug carriers for local chemotherapy [24].

As said, CNHs are good drug carriers and non-covalent interactions thorough bio-modifications improve their ability in the drug delivery systems. On the other hand, the $\mathrm{Na}^{+}$cation present in blood and benzene is an aromatic molecule (most of biological molecules have aromatic parts). In the present study, a $\mathrm{C}_{102} \mathrm{H}_{30}$ graphene sheet with the $C_{2 v}$ symmetry has been rolled up by computational quantum chemistry methods to construct bent graphenes as curved surfaces of CNHs with the same number of atoms. The non-covalent cation- $\pi$ interactions of the $\mathrm{Na}^{+}$cation together with the $\pi-\pi$ stacking interactions of benzene with this graphene sheet and, on the inner and outer faces of the mentioned bent graphenes have been simultaneously investigated to simulate biomodifications of CNHs as drug carriers.

\section{COMPUTATIONAL METHODS}

Structures of all monomers and complexes were optimized with the Gaussian 09 program package [25] at the M052X/6-31G $(d)$ level of theory. The potential energy surface (PES) relaxed scans with keyword opt=modredundant were performed to obtain bent structures. In fact, distances between terminal hydrogen atoms of graphene (in the bending direction) were considered as additional coordinates and kept constant during 100 step-by-step optimizations. In each step, the mentioned distances were reduced by $0.1 \AA$ to obtain the corresponding bent structure. Thus, in each step the optimized bent structure has a slightly larger curvature than the similar optimized structure in the previous step. Also, it should be noted that the terminal $\mathrm{C}-\mathrm{H}$ bond lengths of all structures (in the bending direction) were frozen during all step-by-step optimizations.

Zhao and Truhlar proposed that the M05-2X functional compensates the deficiencies of other hybrid functionals by incorporating improved treatment of the spin kinetic energy density in both exchange and correlation functionals [26]. The diamagnetic and paramagnetic effects of ring currents related to aromaticity and anti-aromaticity can be evaluated by the nucleus independent chemical shift (NICS) [27, 28] criterion. The NMR calculations have been performed at the M05-2X/6$31 \mathrm{G}(d)$ level of theory using the gauge independent atomic orbital (GIAO) method [29].

\section{RESULTS AND DISCUSSION}

To investigate effects of the graphene curvature on mutual cation- $\pi$ and $\pi-\pi$ stacking interactions, a $\mathrm{C}_{102} \mathrm{H}_{30}$ graphene sheet with the $C_{2 v}$ symmetry has been constructed using the HyperChem 7.1 software [30]. To generate bent graphenes, potential energy surface (PES) relaxed scans with keyword opt=modredundant were performed and cation- $\pi$ interactions of the $\mathrm{Na}^{+}$cation and $\pi-\pi$ stacking interactions of benzene with graphene, and on the inner and outer faces of central rings of bent graphenes were concurrently studied (Fig. 1). A curvature parameter $(\mathcal{O})$ is introduced as below to distinguish bent structures

$$
\supset=\operatorname{Ln}\left(d_{0} / d\right)
$$

In this equation, $d_{0}$ is the distance between terminal hydrogen atoms of graphene and $d$ is the distance between terminal hydrogen atoms of bent graphenes. Thus, each bent graphene is compared with graphene and an increase in $\supset$ is consistent 

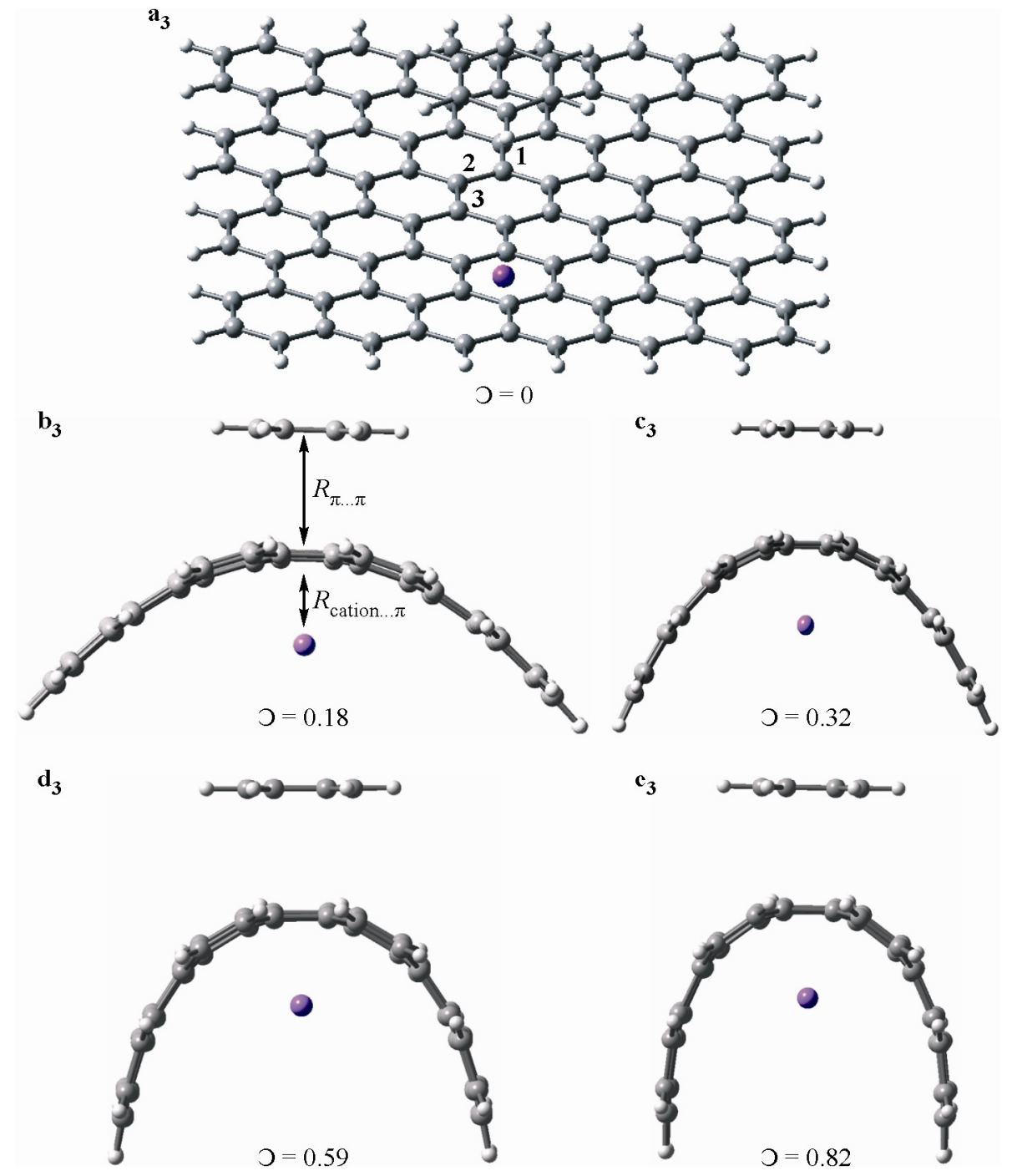

Fig. 1. Presentation of the ternary complexes $\mathbf{a}_{3}-\mathbf{e}_{3}$ together with the corresponding curvature parameters.

with an increase in the curvature of bent graphenes. In fact, the curvature parameter $\supset$ for complexes $\mathbf{a}_{1}, \mathbf{a}_{2}$, and $\mathbf{a}_{3}$ is 0.00 , for complexes $\mathbf{b}_{\mathbf{1}}, \mathbf{b}_{\mathbf{2}}$, and $\mathbf{b}_{\mathbf{3}} 0.18$, for complexes $\mathbf{c}_{\mathbf{1}}, \mathbf{c}_{\mathbf{2}}$, and $\mathbf{c}_{\mathbf{3}} 0.32$, for complexes $\mathbf{d}_{\mathbf{1}}, \mathbf{d}_{\mathbf{2}}$, and $\mathbf{d}_{\mathbf{3}} 0.59$, and finally, for complexes $\mathbf{e}_{1}, \mathbf{e}_{2}$, and $\mathbf{e}_{3}$ 0.82. It should be noted that $\supset$ increases from the letter $\mathbf{a}$ to the letter $\mathbf{e}$ in all notations $\mathbf{a}_{1}-\mathbf{e}_{1}, \mathbf{a}_{2}-\mathbf{e}_{2}$, and also $\mathbf{a}_{3}-\mathbf{e}_{3}$ which are used to distinguish complexes in this study. Typical structures of complexes $\mathbf{a}_{3}-\mathbf{e}_{3}$ are shown in Fig. 1 to illustrate the degree of curvature in all complexes.

Energy data. The binding energy of each ternary complex was computed as a difference between its energy and the energies of isolated components which build the structure. Results indicate that the binding energies of the benzenegraphene- $\mathrm{Na}^{+}$and benzene-bent graphene- $\mathrm{Na}^{+}$complexes are in the range 49.27-53.92 kcal $/ \mathrm{mol}^{-1}$. Moreover, the binding energies of binary graphene- $\mathrm{Na}^{+}$and bent graphene- $\mathrm{Na}^{+}$complexes are in the range $43.18-49.05 \mathrm{kcal} / \mathrm{mol}^{-1}$. On the other hand, the binding energies of binary benzene-graphene and benzene-bent graphene complexes are in the range $3.47-5.19 \mathrm{kcal} / \mathrm{mol}^{-1}$. Thus, attractive interactions between the $\mathrm{Na}^{+}$cation and the inner face of graphene or bent graphenes play an important role in the magnitude of the binding energies of benzene-graphene- $\mathrm{Na}^{+}$and benzene-bent graphene- $\mathrm{Na}^{+}$ complexes.

Results presented in Table 1 show that the graphene curvature firstly leads to a decrease in the binding energies of the benzene-graphene- $\mathrm{Na}^{+}$and benzene-bent graphene- $\mathrm{Na}^{+}$complexes. However, the interactions of more curved graphenes 
TABLE 1. Binding Energy Values of the Binary Complexes $\mathbf{a}_{1}-\mathbf{e}_{1}$ and $\mathbf{a}_{2}-\mathbf{e}_{2}$ and the Ternary Complexes $\mathbf{a}_{3}-\mathbf{e}_{3}\left(\mathrm{kcal} / \mathrm{mol}^{-1}\right)$ Together with Curvature Parameters

\begin{tabular}{c|c||c|c||c|c|c}
\hline Complex* & $-\Delta E$ & Complex** & $-\Delta E$ & Complex*** & $-\Delta E$ & $\square$ \\
\hline $\mathbf{a}_{\mathbf{1}}$ & 5.19 & $\mathbf{a}_{\mathbf{2}}$ & 43.18 & $\mathbf{a}_{\mathbf{3}}$ & 49.78 & 0.00 \\
$\mathbf{b}_{\mathbf{1}}$ & 3.77 & $\mathbf{b}_{\mathbf{2}}$ & 44.07 & $\mathbf{b}_{\mathbf{3}}$ & 49.27 & 0.18 \\
$\mathbf{c}_{\mathbf{1}}$ & 4.25 & $\mathbf{c}_{\mathbf{2}}$ & 44.96 & $\mathbf{c}_{\mathbf{3}}$ & 49.94 & 0.32 \\
$\mathbf{d}_{\mathbf{1}}$ & 3.48 & $\mathbf{d}_{\mathbf{2}}$ & 48.69 & $\mathbf{d}_{\mathbf{3}}$ & 51.05 & 0.59 \\
$\mathbf{e}_{\mathbf{1}}$ & 3.47 & $\mathbf{e}_{\mathbf{2}}$ & 49.05 & $\mathbf{e}_{\mathbf{3}}$ & 53.92 & 0.82
\end{tabular}

* Refer to benzene-graphene and benzene-bent graphene.

** Refer to graphene- $\mathrm{Na}^{+}$and bent graphene- $\mathrm{Na}^{+}$.

*** Refer to benzene-graphene- $\mathrm{Na}^{+}$and benzene-bent graphene- $\mathrm{Na}^{+}$.

with the $\mathrm{Na}^{+}$cation and benzene lead to larger binding energies for the benzene-bent graphene- $\mathrm{Na}^{+}$complexes in comparison with the benzene-graphene- $\mathrm{Na}^{+}$complex. The order of binding energies for the above mentioned ternary complexes is $\mathbf{b}_{3}<\mathbf{a}_{3}<\mathbf{c}_{3}<\mathbf{d}_{3}<\mathbf{e}_{3}$. On the other hand, the order of binding energies in the graphene- $\mathrm{Na}^{+}$and bent graphene- $\mathrm{Na}^{+}$ complexes is $\mathbf{a}_{2}<\mathbf{b}_{2}<\mathbf{c}_{2}<\mathbf{d}_{2}<\mathbf{e}_{2}$. Also, the order of binding energies in the benzene-graphene and benzene-bent graphene complexes is $\mathbf{e}_{\mathbf{1}}<\mathbf{d}_{\mathbf{1}}<\mathbf{b}_{\mathbf{1}}<\mathbf{c}_{\mathbf{1}}<\mathbf{a}_{\mathbf{1}}$. As can be seen, unlike ternary complexes, $\pi-\pi$ stacking interactions of benzene with more curved graphenes lead to small binding energies for the benzene-bent graphene complexes in comparison with the benzenegraphene complex. Results of binding energies in the binary and ternary complexes demonstrate that the order of binding energies in the ternary complexes is mostly consistent with the order of binding energies in the binary graphene- $\mathrm{Na}^{+}$and bent graphene- $\mathrm{Na}^{+}$complexes. The only difference between order of binding energies of the ternary complexes and these binary complexes comes from $\pi-\pi$ stacking interaction of benzene with graphene. As said, the binding energy of complex $\mathbf{a}_{2}$ is lower than that of complex $\mathbf{b}_{2}$. However, the binding energy of the benzene-graphene complex $\mathbf{a}_{1}$ is higher than that of complex $\mathbf{b}_{1}$ and thus, the $\pi-\pi$ stacking interaction of benzene with graphene in the ternary complex $\mathbf{a}_{3}$ leads to a higher binding energy as compared to the complex $\mathbf{b}_{\mathbf{3}}$. This finding shows that although $\pi-\pi$ stacking is a weak interaction, it can influence the order of binding energies of CNHs complexes, which involve both $\pi-\pi$ stacking and cation- $\pi$ interactions. Therefore, $\pi-\pi$ stacking interactions can change the drug delivery abilities of such systems.

Structural parameters. The central ring of graphene was considered as a reaction center to study the effects of the graphene curvature on the structural parameters of bent graphenes and ternary complexes. The equilibrium distance between the $\mathrm{Na}^{+}$cation and the central ring of graphene or bent graphene in ternary complexes is denoted as $\mathrm{R}_{\text {cation... }}$. Also, the equilibrium distance between the center of benzene and the central ring of graphene or each bent graphene is symbolized as $\mathrm{R}_{\pi \ldots \pi}$. All structural parameters are presented in Table 2. As can be seen, $\mathrm{R}_{\text {cation } \ldots \pi}$ values are smaller than $\mathrm{R}_{\pi \ldots \pi}$ ones, which corresponds to the attractive nature of cation- $\pi$ interactions in these complexes. However, the results show that both $\mathrm{R}_{\text {cation... }}$ and $\mathrm{R}_{\pi \ldots \pi}$ values in the ternary complexes increase in the following order: $\mathbf{a}_{3}<\mathbf{b}_{\mathbf{3}}<\mathbf{c}_{\mathbf{3}}<\mathbf{d}_{\mathbf{3}} \cong \mathbf{e}_{3}$. Therefore, the graphene curvature leads to an increase in distances of the $\mathrm{Na}^{+}$cation and benzene from the inner and outer faces of the central rings of bent graphenes, respectively.

TABLE 2. Most Important Structural Parameters $(\AA)$ of the Ternary Complexes $\mathbf{a}_{3}-\mathbf{e}_{3}$

\begin{tabular}{c|c|c|c|c||c|c|c|c|c}
\hline Complex & $\mathrm{C} 1-\mathrm{C} 2$ & $\mathrm{C} 2-\mathrm{C} 3$ & $\mathrm{R}_{\text {cation } \ldots \pi}$ & $\mathrm{R}_{\pi \ldots \pi}$ & Complex & $\mathrm{C} 1-\mathrm{C} 2$ & $\mathrm{C} 2-\mathrm{C} 3$ & $\mathrm{R}_{\text {cation } \ldots \pi}$ & $\mathrm{R}_{\pi \ldots \pi}$ \\
\hline $\mathbf{a}_{3}$ & 1.4212 & 1.4325 & 2.274 & 3.447 & $\mathbf{d}_{3}$ & 1.4184 & 1.4275 & 2.390 & 3.482 \\
$\mathbf{b}_{3}$ & 1.4169 & 1.4235 & 2.314 & 3.469 & $\mathbf{e}_{3}$ & 1.4187 & 1.4283 & 2.458 & 3.480 \\
$\mathbf{c}_{3}$ & 1.4176 & 1.4252 & 2.333 & 3.479 & & & & &
\end{tabular}


The results indicate that there are similar bond lengths in the central ring of graphene and bent graphenes. Thus, only two bond lengths (named $\mathrm{C} 1-\mathrm{C} 2$ and $\mathrm{C} 2-\mathrm{C} 3$ ) were measured. As can be seen in Table 2, both $\mathrm{C} 1-\mathrm{C} 2$ and $\mathrm{C} 2-\mathrm{C} 3$ bond lengths firstly decrease from the complex $\mathbf{a}_{\mathbf{3}}$ to the complex $\mathbf{b}_{\mathbf{3}}$ and then these bond lengths increase from the complex $\mathbf{b}_{\mathbf{3}}$ to the complex $\mathbf{e}_{3}$. Moreover, these bond lengths in complex $\mathbf{b}_{\mathbf{3}}$ have the smallest values. On the other hand, the complex $\mathbf{b}_{\mathbf{3}}$ has the smallest binding energy in comparison with those of other complexes. In fact, the graphene curvature leads to changes in $\mathrm{C}-\mathrm{C}$ bond lengths in the central rings of bent graphenes. Therefore, these structural changes influence the magnitude of simultaneous interactions of the $\mathrm{Na}^{+}$cation and benzene with bent graphenes.

Charge transfer analysis. To relate the energy data on the ternary complexes to the charge transfer effects, Mulliken atomic charges on the $\mathrm{Na}^{+}$cation and the sum of atomic charges of benzene, graphene, and bent graphenes (in e) in the above mentioned complexes were calculated at the $\mathrm{M} 05-2 \mathrm{X} / 6-31+\mathrm{G}(d, p)$ level of theory. These results are presented in Table 3. As can be observed, the positive atomic charge of the $\mathrm{Na}^{+}$cation in the ternary complexes is decreased in comparison with that of the free cation (before complexation). Instead, the formation of ternary complexes is accompanied by an increase in the sum of atomic charges on benzene, graphene, and bent graphenes. Table 3 shows that the sum of positive atomic charges on $\mathrm{H}$ atoms of benzene in the ternary complexes is increased (as compared to 0.5897 e for free benzene). Also, the sum of negative atomic charges on benzene $\mathrm{C}$ atoms in the ternary complexes is increased (as compared to $0.5897 \mathrm{e}$ for free benzene). Additionally, the order of the sum of atomic charges on benzene in the ternary complexes is $\mathbf{a}_{3}<\mathbf{b}_{3}<\mathbf{c}_{\mathbf{3}}<\mathbf{d}_{\mathbf{3}}<\mathbf{e}_{3}$. Correlations between the sum of atomic charges on benzene and the binding energies of the ternary complexes are depicted in Fig. 2. As can be seen, the graphene curvature and an increase in the sum of atomic charges on benzene during the formation of the ternary complexes firstly leads to a decrease and then an increase in the binding energies. In fact, although the sum of atomic charges on benzene in the complex $\mathbf{b}_{\mathbf{3}}$ is larger than in the complex $\mathbf{a}_{3}$, the binding energy of the complex $\mathbf{a}_{\mathbf{3}}$ is larger than that of the complex $\mathbf{b}_{\mathbf{3}}$. Therefore, in complexes formed from bent graphenes which

TABLE 3. Mulliken Atomic Charges (e) on Selected Atoms and the Sum of Atomic Charges on the Components of Ternary Complexes $\mathbf{a}_{3}-\mathbf{e}_{3}$

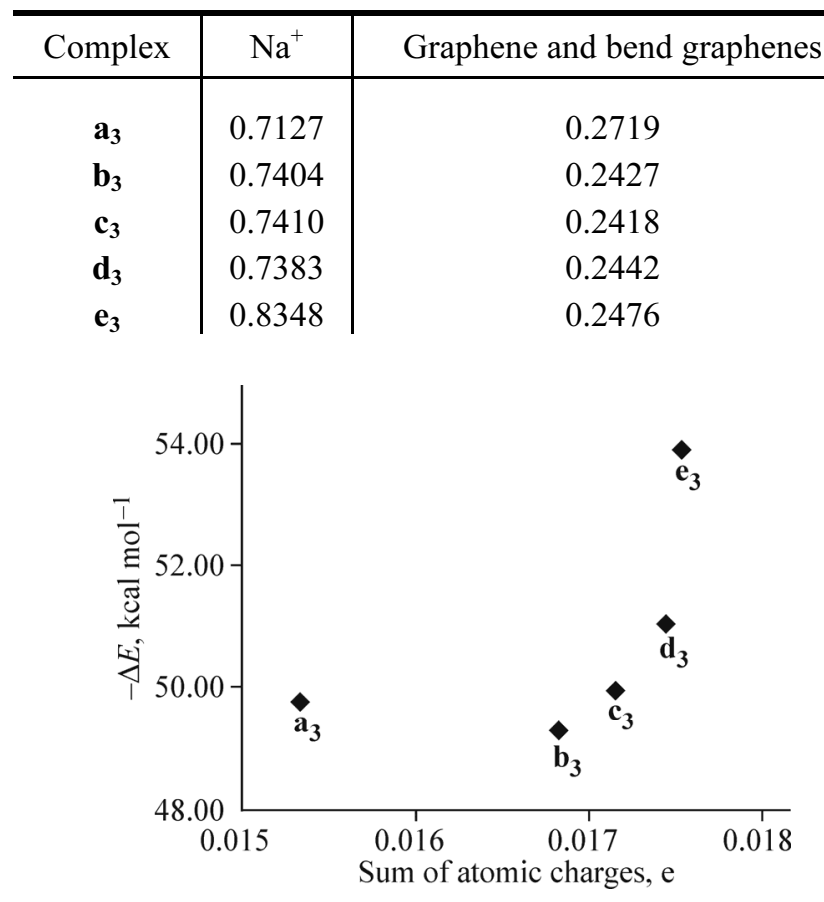

Fig. 2. Correlations between the sum of Mulliken atomic charges on benzene and the binding energies of the ternary complexes.

\begin{tabular}{|c|c|c}
\hline Benzene & H atoms of benzene & C atoms of benzene \\
\hline 0.0153 & 1.0538 & -1.0385 \\
0.0168 & 1.0577 & -1.0409 \\
0.0172 & 1.0580 & -1.0409 \\
0.0174 & 1.0583 & -1.0408 \\
0.0175 & 1.0584 & -1.0409
\end{tabular}

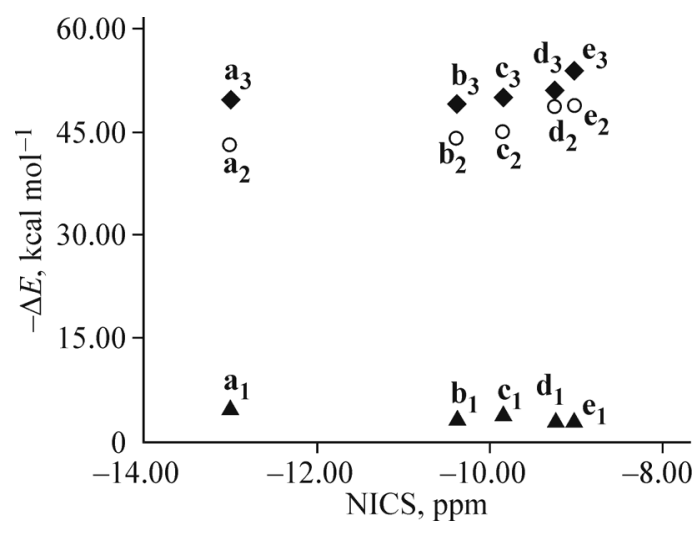

Fig. 3. Binding energies of the complexes $\mathbf{a}_{\mathbf{1}}-\mathbf{e}_{\mathbf{1}}$ $(\boldsymbol{\Delta}), \mathbf{a}_{2}-\mathbf{e}_{2}(\bullet)$, and $\mathbf{a}_{3}-\mathbf{e}_{3}(\bullet)$ against $\operatorname{NICS}(1)$ values above the central rings of graphene and bent graphenes. 
encompass weak interactions such as $\pi-\pi$ stacking, the effects of charge transfer and variations of charges on different ring atoms during complexation may play an important role in the magnitude of binding energies. However, as said, the structural changes in bent graphene in comparison with graphene are essential in this case.

NMR calculations. The NMR calculations have been performed at the M05-2X/6-31G(d) level of theory using the GIAO method. NICS(0) at the center and NICS(1) at $1 \AA$ above the central rings of the graphene sheet and bent graphenes were calculated. The results prove that the rolling of the graphene sheet leads to a decrease in these values. In fact, the rolling of the planar graphene sheet leads to changes in the $\pi$ electron clouds of bent graphenes. The NICS $(0)$ values reveal the effects of $\sigma$ bonds at rings, while the NICS(1) ones reflect the $\pi$ bond effects on the rings. Therefore, NICS(1) is a better criterion than $\operatorname{NICS}(0)$ and stands for the $\pi$ aromaticity of the rings. The binding energies of all the binary and ternary complexes against NICS(1) values at the central rings of graphene and bent graphenes are shown in Fig. 3 . As can be observed in Fig. 3 ( $\boldsymbol{\Delta}$ and $\bullet$ ), a decrease in the aromaticity of the central rings of bent graphenes, which is caused by the graphene curvature, is accompanied by a decrement/increment of binding energies of the benzene-bent graphene/bent graphene- $\mathrm{Na}^{+}$complexes. On the other hand, as can be seen in Fig. $3(\diamond)$, a decrease in the aromaticity of the central rings of bent graphenes goes firstly with a decrement of binding energies of the ternary benzene-bent graphene-Na ${ }^{+}$complexes, which is consistent with the behavior of the binary benzene-bent graphene complexes. Then, the binding energies are increased by a decrease in the aromaticity of the central rings of bent graphenes. These results imply that although $\pi-\pi$ stacking is a weak interaction, it can manipulate the overall behavior of the complexes, which encompass the simultaneous cation- $\pi$ and $\pi-\pi$ stacking interactions, and can influence the drug delivery abilities of such systems.

\section{CONCLUSIONS}

The graphene curvature leads to structural changes in the rings of bent graphenes as curved surfaces of CNHs, which influences the magnitude of biomodifications of these structures through $\pi-\pi$ stacking and cation- $\pi$ interactions. Biomodifications of bent graphenes through the above mentioned interactions are accompanied by an increase in the sum of atomic charges on benzene and bent graphenes. Also, the positive atomic charge of the $\mathrm{Na}^{+}$cation in the ternary complexes is decreased in comparison with that of the isolated cation. The results reveal that the orders of binding energies of the $\mathrm{CNH}$ complexes involve both $\pi-\pi$ stacking and cation- $\pi$ interactions, and thus, their drug delivery properties could be influenced by weak $\pi-\pi$ stacking interactions.

The author thanks the vice-chancellor of research and technology at university of Zabol for financial supports.

\section{REFERENCES}

1. S. Iijima, M. Yudasaka, R. Yamada, et al. Chem Phys Lett., 1999, 309, 165-170.

2. S. Iijima and T. Ichihashi. Nature, 1993, 363, 603-605.

3. S. Utsumi, J. Miyawaki, H. Tanaka, et al. J. Phys. Chem. B, 2005, 109, 14319-14324.

4. M. Harada, T. Inagaki, S. Bandow, et al. Carbon, 2008, 46, 766-772.

5. R. Yuge, M. Yudasaka, K. Yoyama, et al. Carbon, 2012, 50, 1925-1932.

6. E. Bekyarova, K. Murata, M. Yudasaka, et al. J. Phys. Chem. B, 2003, 107, 4681-4684.

7. Y. Hattori, H. Hanoh, F. Okino, et al. J. Phys. Chem. B, 2004, 108, 9614-9618.

8. C. M. Yang, Y. J. Kim, and M. Endo. J. Am. Chem. Soc., 2007, 129, 20/21.

9. A. Izadi-Najafabadi, T. Yamada, D. N. Futaba, et al. ACS Nano, 2011, 5, 811-819.

10. R. Yuge, T. Manako, K. Nakahara, et al. Carbon, 2012, 50, 5569-5573.

11. J. Zhang, J. Lei, C. Xu, et al. Anal. Chem., 2010, 82, 1117-1122.

12. T. Murakami, K. Ajima, J. Miyawaki, et al. Mol. Pharm., 2004, 1, 399-405.

13. K. Ajima, M. Yudasaka, T. Murakami, et al. Mol. Pharm., 2005, 2, 475-480. 
14. M. Zhang, T. Murakami, K. Ajima, et al. Proc. Natl. Acad. Sci. USA, 2008, 105, 14773-14778.

15. M. F. Zhang, M. Yudasaka, K. Ajima, et al. ACS Nano, 2007, 1, 265-272.

16. A. S. D. Sandanayaka, O. Ito, M. F. Zhang, et al. Adv. Mater., 2009, 21, 4366-4371.

17. G. Mountrichas, T. Ichihashi, S. Pispas, et al. J. Phys. Chem. C, 2009, 113, 5444-5449.

18. W. Huang, J. F. Zhang, H. C. Dorn, et al. Bioconjugate Chem., 2011, 22, 1012-1016.

19. X. B. Fan, J. Tan, and G. L. Zhang. Nanotechnology, 2007, 18, 1-6.

20. J. Miyawaki, M. Yudasaka, T. Azami, et al. ACS Nano, 2008, 2, 213-226.

21. K. Ajima, M. Yudasaka, T. Murakami, et al. Mol. Pharm, 2005, 2, 475-480.

22. K. Ajima, T. Murakami, Y. Mizoguchi, et al. ACS Nano, 2008, 2, 2057-2064.

23. J. X. Xu, M. Yudasaka, S. Kouraba, et al. Chem. Phys. Lett., 2008, 461, 189-192.

24. T. Murakami, H. Sawada, G. Tamura, et al. Nanomedicine, 2008, 3, 453-463.

25. M. J. Frisch, G. W. Trucks, H. B. Schlegel, et al. Gaussian 09, Revision A.1. Gaussian, Inc., Wallingford CT, 2009.

26. Y. Zhao, N. E. Schultz, and D. G. Truhlar. J. Chem. Theor. Comput., 2006, 2, 364-382.

27. P. V. R. Schleyer, C. Maerker, and A. Dransfeld. J. Am. Chem. Soc., 1996, 118, 6317/6318.

28. Z. Chen, C. S. Wannere, C. Corminboeuf, et al. J. Chem. Rev., 2005, 105, 3842-3888.

29. K. Wolinski, J. F. Hinto, and P. Pulay. J. Am. Chem. Soc., 1990, 112, 8251-8260.

30. HyperChem ${ }^{\circledR}$ for Windows and NT, Hypercube. Inc., Publication HC50-00-04-00 October, 1996. 